\title{
Association between vestibulo-ocular reflex suppression, balance, gait, and fall risk in ageing and neurodegenerative disease: protocol of a one-year prospective follow-up study
}

Karin Srulijes ${ }^{1,2,7^{*}}$, David J. Mack ${ }^{3,4}$, Jochen Klenk ${ }^{2,5}$, Lars Schwickert ${ }^{2}$, Espen A. F. Ihlen ${ }^{6}$, Michael Schwenk ${ }^{2}$, Ulrich Lindemann ${ }^{2}$, Miriam Meyer ${ }^{1}$, Srijana K.C. , Markus A. Hobert ${ }^{1,7}$, Kathrin Brockmann ${ }^{1,7}$, Isabel Wurster ${ }^{1,7}$, Jörn K. Pomper ${ }^{3}$, Matthis Synofzik ${ }^{1,7}$, Erich Schneider ${ }^{8}$, Uwe $\| l g^{3}$, Daniela Berg ${ }^{1,7}$, Walter Maetzler ${ }^{1,7}$ and Clemens Becker ${ }^{2}$

\begin{abstract}
Background: Falls frequency increases with age and particularly in neurogeriatric cohorts. The interplay between eye movements and locomotion may contribute substantially to the occurrence of falls, but is hardly investigated. This paper provides an overview of current approaches to simultaneously measure eye and body movements, particularly for analyzing the association of vestibulo-ocular reflex (VOR) suppression, postural deficits and falls in neurogeriatric risk cohorts. Moreover, VOR suppression is measured during head-fixed target presentation and during gaze shifting while postural control is challenged. Using these approaches, we aim at identifying quantitative parameters of eye-head-coordination during postural balance and gait, as indicators of fall risk.

Methods/Design: Patients with Progressive Supranuclear Palsy (PSP) or Parkinson's disease (PD), age- and sexmatched healthy older adults, and a cohort of young healthy adults will be recruited. Baseline assessment will include a detailed clinical assessment, covering medical history, neurological examination, disease specific clinical rating scales, falls-related self-efficacy, activities of daily living, neuro-psychological screening, assessment of mobility function and a questionnaire for retrospective falls. Moreover, participants will simultaneously perform eye and head movements (fixating a head-fixed target vs. shifting gaze to light emitting diodes in order to quantify vestibulo-ocular reflex suppression ability) under different conditions (sitting, standing, or walking). An eye/head tracker synchronized with a 3-D motion analysis system will be used to quantify parameters related to eye-head-coordination, postural balance, and gait. Established outcome parameters related to VOR suppression ability (e.g., gain, saccadic reaction time, frequency of saccades) and motor related fall risk (e.g., step-time variability, postural sway) will be calculated. Falls will be assessed prospectively over 12 months via protocols and monthly telephone interviews.

(Continued on next page)
\end{abstract}

\footnotetext{
* Correspondence: karin.srulijes@uni-tuebingen.de

'Department of Neurodegeneration, Hertie Institute for Clinical Brain

Research, University of Tuebingen, Tuebingen, Germany

2Department of Geriatrics and Clinic of Geriatric Rehabilitation,

Robert-Bosch-Hospital, Stuttgart, Germany

Full list of author information is available at the end of the article
} 
(Continued from previous page)

Discussion: This study protocol describes an experimental setup allowing the analysis of simultaneously assessed eye, head and body movements. Results will improve our understanding of the influence of the interplay between eye, head and body movements on falls in geriatric high-risk cohorts.

Keywords: Vestibulo-ocular reflex suppression, VOR, Falls, Postural stability, Gait, Balance, Neurodegeneration, Parkinson's Disease, Parkinsonism, Older persons

\section{Background}

Worldwide, the number of persons aged 60 and older is expected to almost triple from 841 million in 2013 to over two billion in 2050 [1]. Since falls are more common [2] and their consequences often more severe [3] in the older population, this demographic development will be accompanied by an increased frequency of falls. The according personal, social and economic burden will have a strong impact on society. Many risk factors for falls like gait and balance deficits [2] have been identified in the older population, but the contribution of failures in the interplay between oculo- and locomotion (especially combined head and body movement) to the risk of falls has not yet been studied in detail. This is surprising given that visual exploration of the environment while standing or walking is regularly required during daily life (e.g. crossing a street), and deficits in the oculomotor system may have impacts on the performance of the locomotor system, and vice versa. Hollands and Marple-Horvat provided evidence for the interaction of the oculo- and locomotor systems during a visually guided stepping task [4]. They found that any variation in stance duration was accompanied by a variation in timing of saccade onset with the interval between saccade onset and foot lift being constant. Glasauer and colleagues described another interaction between eye movements and postural control: not only retinal slip provides a relevant feedback for postural control, but also slow eye movements [5].

Importantly, different tasks require different oculomotor strategies. During normal ambulation and head movement, the vestibulo-ocular reflex (VOR) stabilizes gaze, and therefore helps to keep the image of the environment stable on the retina. This reflex enables antagonistic eye and head movements. In contrast, maintaining gaze on a moving object or shifting gaze to a new object of interest e.g., while walking on the sidewalk and suddenly fixating a car driving towards oneself, requires the eyes and the head to move into the same direction. For the proper performance of these agonistic concerted movements, the suppression of the vestibulo-ocular reflex (VOR suppression) is inevitable [6, 7]. The VOR suppression enables the guidance of the eyes into the direction of the head during self-generated directional head movements, and can be performed either very fast (while gaze shifting, see examples above) or smoothly (e.g., when walking and pursuing a car driving past). During natural visual exploration in ecologically valid environments $22 \%$ of eye movements do not compensate for the head movements, but are synergistic [8].

Two different forms of eye movements are used to change gaze, which is to move the eye in space to follow a moving target - smooth pursuit; or to redirect the eye to a new target of interest - saccades. As a consequence, the image of the target is presented on the fovea (area of highest resolution) in both cases. Saccades are very fast gaze shifts which rapidly place an object of interest onto the fovea, whereas smooth pursuit is slow. Head movements can be added to both forms of eye movements to achieve a wider range of motion [9]. The underlying neuronal systems are different for fast eye/head movements and smooth eye/head movements which illustrate the need to analyze VOR suppression separately for both types of gaze changes.

\section{Vestibulo-ocular reflex suppression during smooth eye-head pursuit}

Two separate mechanisms have been discussed to enable VOR suppression during eye-head pursuit, that is to enable to overcome the vestibular drive that would move the eyes away from the target of interest. The first is the cancellation of the VOR by a smooth-pursuit signal based on a cerebro-ponto-cerebellar pathway [9]. The second mechanism is a partial, parametric reduction of VOR gain (eye velocity/head velocity) [10].

Three types of neurons in the vestibular nuclei are probably involved in both processes: position-vestibularpause neurons, vestibular-only neurons and flocculus target or eye-head neurons [10]. Concerning the first mechanism, eye-head neurons seem to modulate their discharge during smooth pursuit and eye-head tracking, by possibly receiving a pursuit signal from the vestibulocerebellum. The second mechanism of reduction of VOR gain seems to be achieved by the attenuation of the discharge of position-vestibularpause neurons. This mechanism might be elicited by mat ching of proprioceptive signals from the neck and the according, expected signals of the neck motor command [11]. These pathways point to the complexity of regulation of the VOR suppression, involving a large network of brain areas and, therefore, being vulnerable to the risk of age- and brain lesion-associated deficits of this movement. 
Vestibulo-ocular reflex suppression during rapid eye-head gaze shifts

Looking down on the pavement to step over a hole is made possible by a highly coordinated fast eye-head movement. To perform such larger eye-head gaze shifts beyond the oculomotor range, saccadic and vestibular signals have to be well coordinated.

A saccade is a rapid eye movement that shifts the line of sight between points of fixation [10]. Controlling circuits involve the superior colliculus, the frontal and parietal eye field and the horizontal and vertical brainstem saccade generators [12].

VOR suppression during rapid eye-head gaze shifts seems to have special characteristics. In healthy individuals, VOR suppression has been shown to antedate head motion, gaze and attention shift [13] (see also Fig. 1). Experiments analyzing the time course of VOR suppression during large eye-head gaze shifts in monkeys found maximal VOR suppression early in the gaze shift and normal VOR gain only near the end [14]. Moreover, VOR suppression seems to be a basic and task-dependent mechanism. Larger saccade amplitudes and gaze shifts are correlated with the strength of VOR suppression $[15,16]$. It has been described, that position-vestibular-pause interneurons of the VOR seem to attenuate their discharge by $75 \%$ or more during head free gaze shift in the monkey [17] but exact mechanisms between saccade generating brainstem circuits and vestibular driven eye movements are still to be investigated [15]. Again, the complex pathway of the human brain necessary to produce VOR suppression during gaze shifting also suggests a high vulnerability of this pathway to age- and disease-related brain pathology. Indeed, impaired VOR suppression has been shown to cause delayed or imprecise obstacle perception, and even relatively unspecific symptoms, such as dizziness, diplopia, blurred vision and unstableness during fast head and body movements [18-20].

\section{Vestibulo-ocular reflex suppression and aging}

There is increasing evidence that age influences the function of VOR performance and suppression. It has been shown that older adults have a decreased ability to enhance and suppress the VOR, compared to young adults [21-23]. Moreover, several studies have shown impaired VOR suppression associated with age-related neurodegenerative diseases, like Parkinson's disease (PD) [24-26] and progressive supranuclear palsy (PSP). PSP is a rare neurodegenerative condition with relatively rapid decline in oculomotor function, and regular occurrence of falls already in the early disease stages [27].

The positive association between intact VOR suppression during vertical smooth head movements and cognitive functioning in PSP patients might be taken as indication that both functions rely on multiple brain regions with the consequence to be similarly affected by widespread degenerative processes [28].

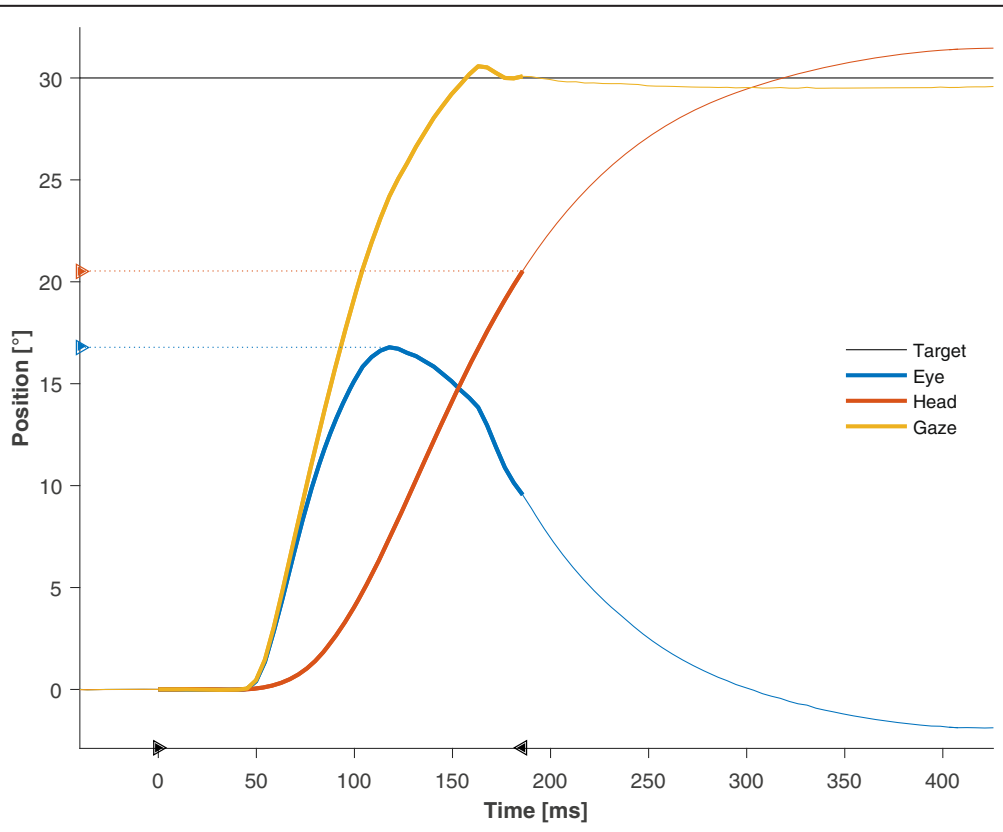

Fig. 1 An example of the time course of a coordinated eye-head movement to a target jump of $30^{\circ}$ consisting of a primary gaze shift (thick lines) For the primary gaze shift, it can be clearly seen that it is mainly dominated by the eye contribution during the first 50 ms. Markers on the $x$-axis indicate the start and end of the gaze shift. Markers on the y-axis show the peak contribution of the eye (blue) and the head (red) to the gaze shift 
Recent analysis of gaze and postural coordination found age-related impairment of saccadic accuracy during postural challenge [29]. In addition, analysis of the timing of eye and body movements revealed interesting relationships. Saccade-to-stepping delays in older persons at high risk of falling may be attributed to longer central nervous system processing time necessary to plan precise foot placements [30].

\section{Vestibulo-ocular reflex suppression in relation to balance deficits and falls}

Accumulating evidence shows a direct association between deficits in VOR suppression and falls. Eighteen older females at increased risk of falls did not suppress the VOR as effectively as 18 matched females at low risk of falling [31]. These high risk patients could not look on the floor while initiating stance from a sitting position and therefore were impaired in observing the ground surface. Di Fabio and colleagues [32] showed in a sample of 38 women with a mean age of 81.6 years that inadequate VOR suppression led to an 18 -fold increased risk for falls, compared to an adequate VOR suppression.

One longitudinal study investigated oculomotor and gait and balance function using the Tinetti balance test [33]. Assessing 53 healthy older individuals annually over a time period of 9 years, the authors found a decline in VOR and VOR suppression performance correlating significantly with a decline in this test.

Stoffregen et al. found in 12 young subjects reduced postural sway variability during target oscillation compared to sway while viewing a stationary target. They stated that postural control is important for the stabilization of the visual system to allow accurate gaze movements [34]. This is interesting, as this study argues for a functional integration of postural control with visual performance. Still, analysis of simultaneously assessed VOR suppression, balance and gait parameters has not been performed yet.

Young and Hollands discovered longer saccadic reaction latencies towards a target in older participants at high risk for falls following target perturbation and required stepping adjustment [35]. Taken together, mutual influence of eye and body movements seems possible and simultaneous analysis challenging eye and body movements in a systematic approach could lead to further understanding of mechanistic aspects of the association of these systems in ageing and neurodegenerative disease.

Although it seems that the association of VOR suppression deficits and falls is mediated by postural and gait deficits, exact mechanistic data on the interaction of eye, head and body movements in older adults with and without specific balance impairments, and its association with falls, is still largely lacking.

\section{Current research questions and how to test them (see Fig. 2)}

(i) Is there a difference in VOR suppression ability performing slow (smooth eye/head tracking with head-fixed target) or fast head movements (rapid eye/head gaze shifts)? Combined measurement with eye/head tracker and a head-fixed target will allow analyzing VOR suppression during slow eye/head pursuit. Measurement with eye/head tracker and stimulus presentation via light emitting diodes will be used to measure the suppression ability during fast eye/head movements.

(ii)Is VOR suppression ability dependent on body position (sitting / standing / walking)? Assessment of VOR suppression ability will be performed in all three body positions.

(iii)Are VOR suppression deficits (during slow eye/head pursuit and rapid gaze shifting) associated with alterations of sway and gait parameters? Synchronized eye/head tracking and 3-D body motion analysis systems will allow the simultaneous assessment of VOR suppression ability and balance and gait parameters (see Fig. 2).

(iv)Are these gait and balance alterations able to predict falls? Prospective falls assessment will be used to analyse the validity of assessed clinical and biomechanical parameters to predict future falls.

(v) Does aging and neurodegeneration have an effect on above listed questions? Assessments will be performed in young and older healthy as well as in neurogeriatric high-risk cohorts for falls to analyse effects of aging and neurodegeneration.

\section{Methods/design}

\section{Study design}

This study has a cross-sectional design to analyze the interplay between vestibulo-ocular reflex suppression, balance and gait, as well as a longitudinal design to assess their effect on future falls. All participants will obtain extensive clinical and quantitative assessment at the Robert-Bosch-Hospital Stuttgart. All procedures are approved by the local ethics committee (University of Tübingen, 602/2012BO1) and are in agreement with the Declaration of Helsinki.

\section{Participants}

Healthy young adults and healthy older adults (age- and sex-matched to the patient cohorts) will be recruited with the support of the office of Sport and Exercise, city of Stuttgart, Germany and the Bosch BKK health insurance.

Progressive Supranuclear Palsy and Parkinson's disease patients will be recruited by movement disorders specialists of the Center for Neurology of the University of Tuebingen. We aim at recruiting 8 infrequent fallers ( $\leq 1$ fall/year) and 


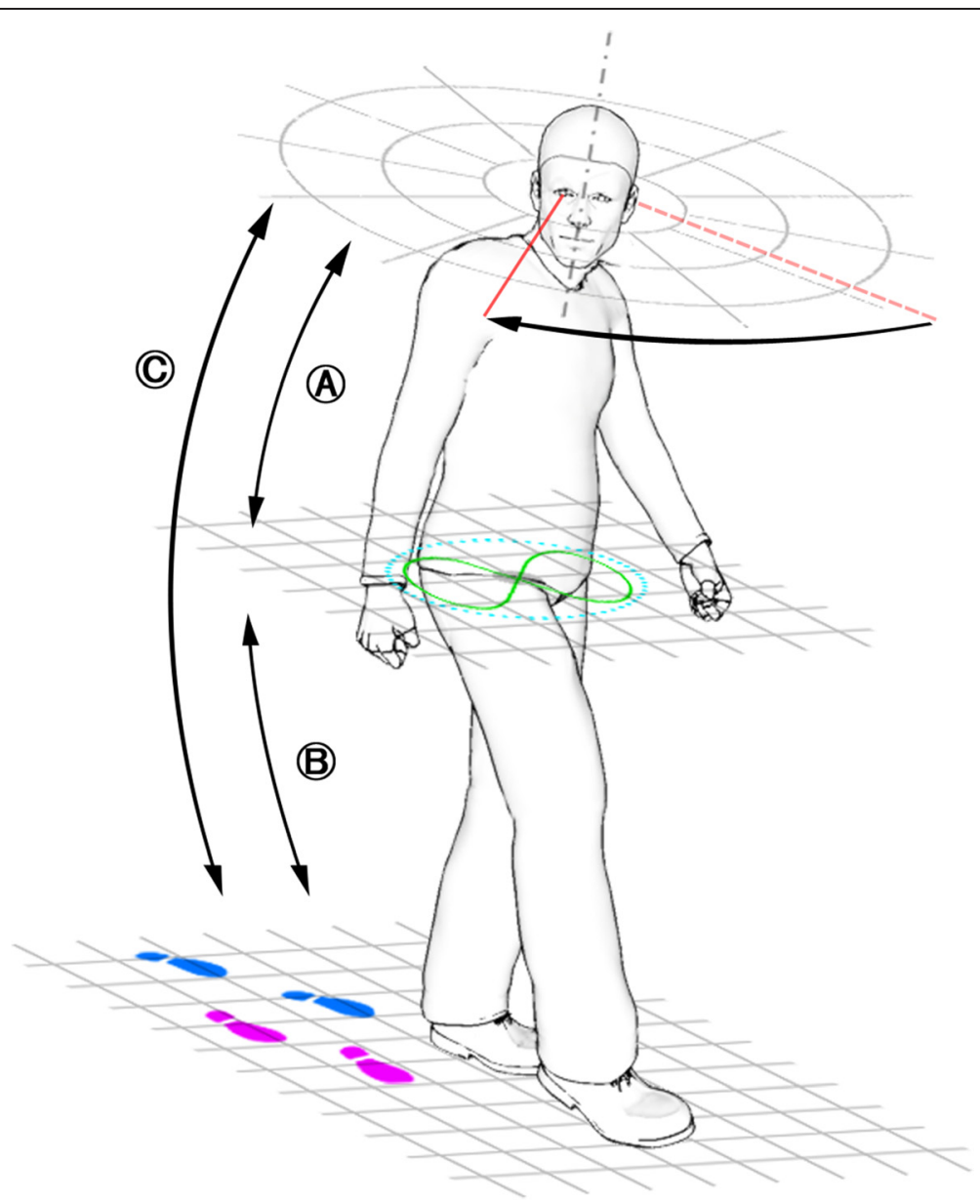

Fig. 2 Schematic illustration of subsequent research questions, showing a subject performing a gaze shift while walking. a Interplay of eye/head movement and balance; $\mathbf{b}$ Interplay of balance and gait; $\mathbf{c}$ Interplay of eye/head movement and gait

8 high frequent fallers ( $\geq 5$ falls/ year) per cohort. Cohort size is based on feasibility (rare disease) and on comparable studies publishing significant results with similar cohort sizes (4 individuals in [4]; 8 individuals in [28]; 15 individuals in [36]).

Inclusion criteria for the patients will be probable or possible diagnosis of PSP [37] or PD (UK Brain Bank criteria [38]), disease stage according to Hoehn and Yahr stage I to IV [39], ability to walk $20 \mathrm{~m}$ with or without walking aid, and global cognitive test (Montreal Cognitive Assessment [MoCA] score $\geq 18$ ).

Exclusion criteria for all cohorts will be neurological di sorders (except the diagnosis of PSP or PD in the patient cohorts), manifest daily relevant dementia, psychiatric di sorders, drug abuse, ophthalmologic disorders, extremity prosthesis, arthritis or musculoskeletal injuries in the past 3 months. As glasses cannot be worn using the eye-tracking device, participants requiring visual correction by glasses stronger than $\pm 3 \mathrm{dpt}$ will not be included in this study. All participants will have to give their written informed consent.

\section{Clinical assessment}

\section{Multifactorial geriatric risk assessment}

Clinical data like sex, age, body mass index as well as medical history (including dose and name of drugs taken, separate listing of drugs affecting the central nervous system) will be assessed. Furthermore, comorbidity will be assessed using the Functional Comorbidity Index [40].

Occurrence of falls during the last week, last month, last 6 and 12 months will be inquired. For the assessment of falls-related self-efficacy, the Short Form of the Falls Efficacy Scale-International (FES-I) will be used. This scale has been shown to be highly related to previous and subsequent falling [41].

Activities of daily living will be assessed by the short form of the questionnaire Late-Life Function and Disability Instrument (LLFDI) [42].

Neuropsychological examination includes assessment of global cognition using the Montreal Cognitive Assessment (MoCA) [43]. Executive dysfunction will be tested using the Trail Making Test parts $A$ and B (TMT A+B) [44]. The use of the Allgemeine Depressions Skala (ADS) [45] 
will help to quantify mood disturbances. Behavioral disturbance will be assessed with the Neuropsychiatric Inventory (NPI) [46].

To assess and compare motor impairment across different neurodegenerative diseases the Unified Multiple System Atrophy Rating Scale (UMSARS) will be used [47].

\section{Disease specific assessment}

Disease severity and symptoms will be assessed by the Progressive Supranuclear Palsy Rating Scale (PSP-RS) [48] and the Unified Parkinson's Disease Rating Scale (MDS- UPDRS) [49].

\section{Mobility-function assessment}

For the assessment of the participants ability to adapt his gait to different tasks like head movement, turning or stair climbing the Dynamic Gait Index (DGI) will be used [50].

\section{Experimental setup (see Fig. 3)}

\section{Eye-head tracking}

A mobile, video-oculography-based eye tracker (EyeSee$\mathrm{Cam}^{\bullet}$, EyeSeeTec GmbH, Fürstenfeldbruck, Germany) will be used to record the participant's eye and head movements. The device is head-mounted with tightly-fitting goggles and a head band to prevent movement induced slip. The eye tracker records eye-in-head movements of the left eye (sampling rate: $220 \mathrm{~Hz}$, spatial resolution: $0.05^{\circ}-0.1^{\circ}$, accuracy: $1^{\circ}$ ). An accelerometer and a gyroscope record the relative head movements. Calibration is done using an integrated laser with a diffraction grating (5-point laser grid). All data is transferred to a laptop via FireWire.

\section{3-D motion analysis}

A six-camera VICON T10 system $\left(\right.$ Vicon $^{\odot}$ Motion Systems Ltd.UK) will be used to record absolute head and body motions. The full body model consists of 15 reflective markers positioned on the head-fixed target helmet (see Fig. 3 - front, top, back), trunk (jugulum, $7^{\text {th }}$ cervical vertebra, $5^{\text {th }}$ lumbal vertebra), arms and feet.

\section{Stimulus presentation LEDs}

Seven light emitting diodes (LEDs) as dot lights will be arranged in front of the participant (see Fig. 3). They will be positioned at $0^{\circ}$ in front of the participant at a distance of $120 \mathrm{~cm}$ and at fixed positions to the left and right side of the participant. The height will be adjusted to the level of each participant's eyes.

The LED will be controlled using a programmable microcontroller. Stimulus presentation time will be $500 \mathrm{~ms}$. Stimulus presentation will be unpredictable in a random but fixed order with varying inter-stimulus-intervals to provoke fast eye-head gaze shifts.

EyeSeeCam ${ }^{\circ}$, the VICON system and the stimulus presentation LEDs will be synchronized via a synchronization signal.

\section{Body-worn sensor}

To record further gait parameters we will use a wearable sensor (Dynaport Hybrid, McRoberts BV, NL) with a

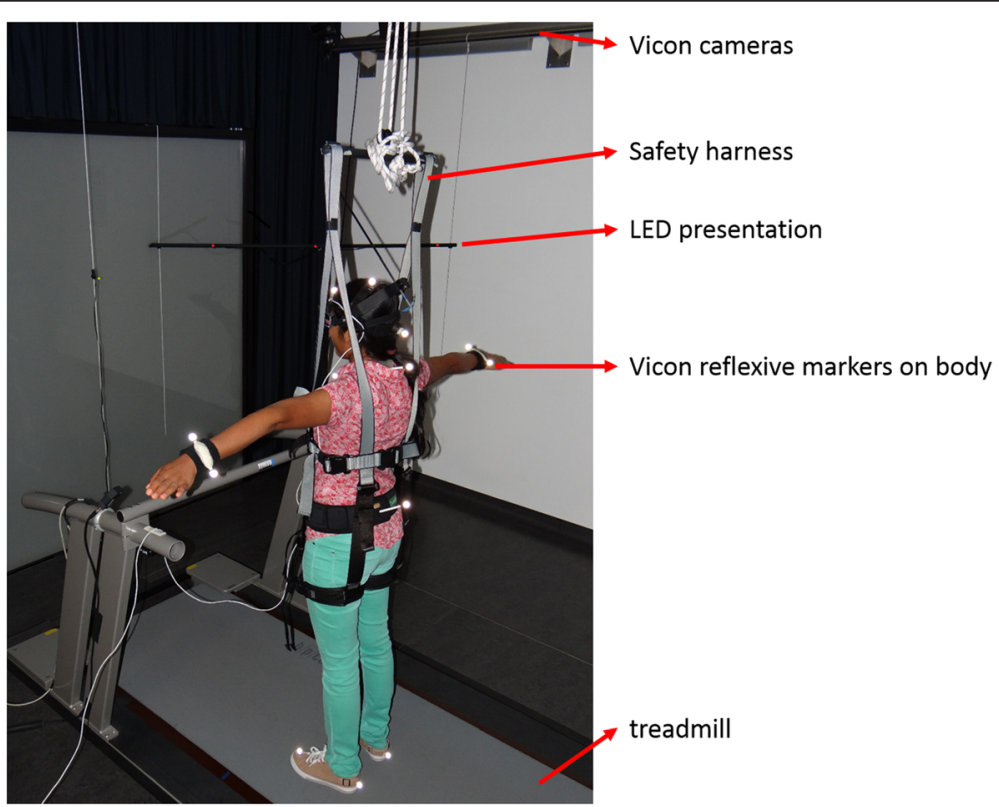

Fig. 3 This photograph shows a doctoral student wearing a part of the experimental setup. The student is standing on the treadmill wearing a safety harness. Vicon reflective markers are placed on central positions for skeleton-fitting of the body. The central LED is positioned at $0^{\circ}$ at a distance of $120 \mathrm{~cm}$. Three LEDs are presented to the right side of the participant (left side not shown here) 
combined triaxial accelerometer (sampling rate $100 \mathrm{~Hz}$; range $\pm 2 \mathrm{~g}$; resolution $\pm 1 \mathrm{mg}$ ) and triaxial gyroscope (range $\pm 100 \%$ s; resolution $\pm 0.0069 \%$ ). The sensor will be worn at the lower back at the height of the second lumbar vertebra, fixed with an elastic belt.

\section{Treadmill}

A treadmill $(\mathrm{h} / \mathrm{p} / \mathrm{cosmos}$ venus; length $200 \mathrm{~cm}$, width $75 \mathrm{~cm}, \mathrm{~h} / \mathrm{p} / \mathrm{cosmos}$ sports medical GmbH, Germany) will be used for the walking paradigm. Participants will be allowed to get accustomed to treadmill walking before the trial start. Comfortable gait speed and fast gait speed will then be estimated and documented in $\mathrm{m} / \mathrm{s}$.

\section{Experimental protocol (see Table 1)}

Prior to each condition and in case of slipping of the eye-tracking system, calibration will be performed.

Subjects will be asked to reduce blinking if possible to avoid data loss in the eye position. Artificial tears will be provided, if necessary.

In all procedures data quality will be visually monitored in real time and additional trials will be completed when needed, to ensure enough trials with good quality for analysis.

\section{Vestibulo-ocular reflex (Head-impulse test, HIT)}

VOR function will be tested first in the sitting condition (see Additional file 1: Video A). First the response to passive yaw head impulses will be investigated [51], which is an unpredictable horizontal head rotation of low amplitude, high acceleration and high peak velocity.

The examiner will be standing behind the participant, asking him to relax the shoulders and neck muscles. The

Table 1 Experimental design to allow simultaneous analysis of vestibulo-ocular reflex, vestibulo-ocular reflex suppression and balance (while standing) and balance and gait (while walking)

\begin{tabular}{llcc}
\hline & Sitting & Standing & Walking \\
\hline Head-impulse test (VOR) passive & $\checkmark$ & & \\
Head-impulse test (VOR) active & $\checkmark$ & $\checkmark$ & $\checkmark$ \\
Smooth eye-head tracking passive & $\checkmark$ & & \\
Smooth eye-head tracking active & $\checkmark$ & $\checkmark$ & $\checkmark$ \\
"Natural" walking & & & $\checkmark$ \\
Fixation-/no-fixation & & $\checkmark$ & $\checkmark$ \\
Rapid eye-head gaze shifts & $\checkmark$ & $\checkmark$ & $\checkmark$ \\
\hline
\end{tabular}

Measurements will be performed in three different conditions: Sitting, Standing, and Walking. Passive $=$ head movement made by examiner. Active $=$ head movement made by subject (important while standing and walking, as the examiner should not influence the subjects balance and gait). VOR = vestibulo-ocular reflex. Smooth eye-head tracking = vestibulo-ocular reflex suppression test with head-fixed target. "natural" walking = treadmill walking without presentation of visual stimuli (control task). Fixation-/no-fixation = standing or walking $30 \mathrm{~s}$. without fixation of a visual target, standing or walking $30 \mathrm{~s}$. while fixation of a visual target. Rapid eye-head gaze shifts = vestibulo-ocular reflex suppression test while rapid gaze shifts participant will be required to continuously fixate the earth-fixed central LED target during the impulses.

\section{Vestibulo-ocular reflex suppression with head-fixed target (VOR S)}

VOR suppression during smooth eye-head tracking will be assessed using a head-fixed target (see Fig. 4). The fixation LED will be positioned $30 \mathrm{~cm}$ in front of the participant's eyes. First, VOR suppression will be tested passively with head movements performed by the examiner standing behind the participant. The participants head will be rotated at approximately $0.2 \mathrm{~Hz}$. The participant will be instructed to continuously fixate the head-fixed target positioned in front of his eyes. The participant's oculomotor performance can be continuously controlled by the examiner by real-time signal observation on the laptop screen.

After the passive VOR suppression performance, active VOR suppression performance (head movement without control by the examiner while fixation of the head-fixed target) will be assessed (see Additional file 2: Video B). The participant will be allowed to practice the active VOR suppression performance to adapt velocity and amplitude to the passive VOR suppression performance. Passive and active VOR suppression performance will be assessed in the horizontal and vertical plane.

This VOR suppression testing will also be performed in the standing and walking condition. Safety will be ensured by the use of a relatively unobtrusive harness. During the standing trials, the participant will be asked to stand relaxed with his hands by his side while performing sinusoidal head movements and fixating the head-fixed LED target. The active VOR suppression will be performed at steady-state gait speed during walking on the treadmill at the previously selected individual comfortable gait speed (see Additional file 3: Video C).

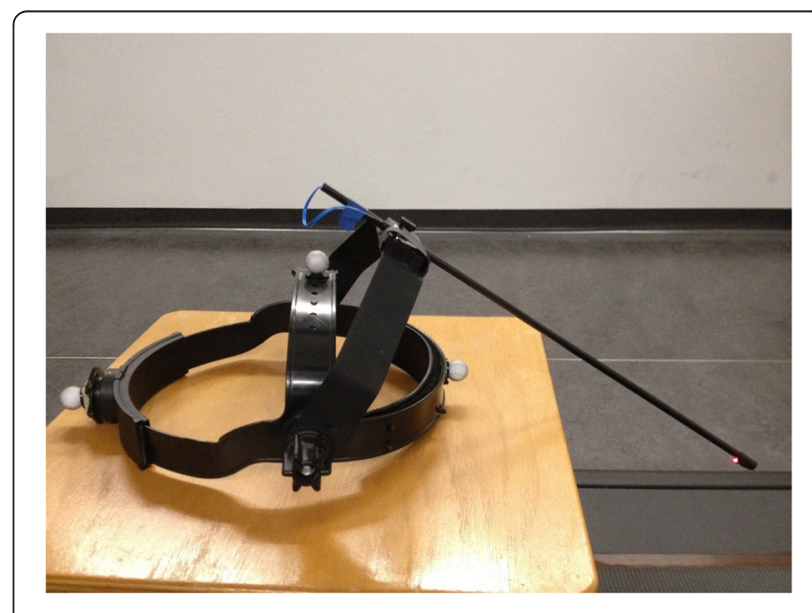

Fig. 4 Custom made head-fixed target with reflective Vicon marker 


\section{Rapid eye-head gaze shifts}

Gaze shifting ability will be tested first in the sitting condition (see Additional file 4: Video D).

The participant will be asked to fixate the central LED $\left(0^{\circ}\right)$. The order of conditions will be fixed to sitting (1.), standing (2.) and walking (3.), as counter-balancing does not seem reasonable due to the limited group size. The participant will be asked to fixate the LEDs "as fast as possible" whenever they appear and after each fixation, re-fixate the central LED.

Safety will again be ensured by the use of the harness. During the standing trials, the participants will be asked to stand relaxed with their hands by their side facing the central LED. Then the gaze shifting will be performed at steady-state gait speed during walking on the treadmill at the previously selected individual comfortable gait speed (see Additional file 5: Video E).

\section{Experimental variables}

\section{Eye and head movements}

Raw data will be processed offline using MATLAB (Matlab R2013b, MathWorks, Natick, MA) software.

Eye position, eye velocity and head velocity will be taken from the values returned by the eye tracker software, which applies an internal blink-removal method and a Gaussian zero-phase low-pass filter to the eye data. All data will be upsampled using a piece-wise cubic Hermite polynomial interpolation to match the sampling rate of the Vicon data $(1 \mathrm{kHz})$. Eye and head accelerations will be computed from head velocity with a central difference method. Head position will be acquired from head velocity using a detrended cumulative sum.

HIT detection Saccades and head impulses will be automatically detected using a two-stage head velocity threshold and a $20 \mathrm{~ms}$ moving standard deviation for eye acceleration. All detected head impulses will be visually inspected and corrected if needed. Valid trials will be determined based on peak head velocity and acceleration as well as eye velocity smoothness, e.g., the standard deviation of the difference of successive velocity samples. Trials in which the overall smoothness exceeds $30^{\circ} \mathrm{s}$ will be excluded, since the pupil is not correctly detected by the eye tracker. At the beginning of each trial, maximum head velocity will have to be lower than $40 \%$. For a trial to be valid, peak head velocity will have to be between $45 \%$ and $500 \%$ and will have to occur within 60 and $200 \mathrm{~ms}$ after onset of the head movement. Peak head acceleration will have to lie between $1.000 \% \mathrm{~s}^{2}$ and $10.000 \% \mathrm{~s}^{2}$. Trials, in which an eye movement of more than $1^{\circ}$ in the same direction as the head movement occurs, will be excluded as anticipatory response.

Over all trials, the following parameters will be determined: Median instantaneous velocity gain (-eye velocity / head velocity) at 40, 60 and $80 \mathrm{~ms}$ after head impulse onset and mean peak head velocity, acceleration and deceleration with corresponding times from onset. To get a measure for the symmetry of the head movement, the signed acceleration amplitude (absolute peak deceleration - absolute peak acceleration) will be computed, which yields values close to 0 for very symmetric movements, but normally is negative since accelerations tend to be higher than decelerations. Head-impulse velocity gain of the VOR will be calculated with a robust ordinary least-squares regression from eye and head velocity [52]. All output parameters will be generated for the left and right side separately.

VOR suppression (VORS) during smooth eye-head tracking with head-fixed target $\mathrm{A}$ head velocity threshold of $10 \%$ will be used for the detection of the VORS period. Saccades will be detected with a $15 \mathrm{~ms}$ moving standard deviation of the eye position. Saccades with amplitudes smaller than $1^{\circ}$, durations less than $5 \mathrm{~ms}$ and peak velocities outside the range of 20 to $1000 \%$ will be considered artefacts and removed. For the horizontal and vertical dimension, saccades will be grouped in the corresponding hemifield (left/right, up/down) and their number, frequency, mean amplitude, duration and peak velocity are computed. VORS quantity will be assessed for each direction with the median position gain (eye position / head position), standard deviation of the eye position and the saccade frequency over the whole period.

In addition, peak head frequency $\left(f_{\text {max }}^{H}\right)$ and the corresponding amplitude $\left(a_{\max }^{H}\right)$ will be derived from the Fourier transform of the head velocity. The amplitude in the Fourier transform of the eye velocity at the peak head frequency $\left(a^{E}\right)$ will be computed and a compensation factor for the VORS will be derived from $C=\left(1-a^{E} / a_{\max }^{H}\right)$ -100. The compensation factor ranges from $0 \%$ at no VORS to $100 \%$ at perfect VORS.

Rapid eye-head gaze shift Gaze position will be computed through linear addition of eye and head position. The target appearance will be externally controlled and the control signal will be send to the Vicon software. To synchronize this signal with the gaze data, participants will have to move their head up and down in the beginning of the gaze shift experiment to get a strong peak in head position in both datasets. The lag between eye tracker and Vicon data will be accordingly corrected using a crosscorrelation procedure. Saccades will be detected similarly as in the VORS trials but with a larger window size of $150 \mathrm{~ms}$ and only saccades with amplitudes of more than $5^{\circ}$ and durations longer than $25 \mathrm{~ms}$ will be considered. For the first detected saccade in each trial that occurred within $2 \mathrm{~s}$ of the target onset, reaction time, robust start and end positions (median position over $100 \mathrm{~ms}$ before/after the 
on-/offset), robust amplitude (robust end position - robust start position) and duration will be computed. To measure the accuracy of the gaze shift, the robust position gain (robust end position / target position) will be determined. To quantify the dynamic properties of the saccades, the main sequence will be computed for duration versus robust amplitude with a robust linear least-squares regression [52].

\section{Body movements}

The gait parameters will be selected on previously established associations with fall risk [53, 54].

3-D Motion capture system (Vicon) Mediolateral displacements of body segments [55] as well as the gait phases and their durations will be calculated. The analysis of dynamic stability at touchdown of the right and left foot will be aimed at using the margin of stability. It is determined as the difference between base of support and extrapolated center of mass (CoM) [56]. The extrapolated CoM can be defined by calculation of the horizontal component of the projection of the CoM to the ground, the horizontal CoM velocity and the distance between CoM and center of ankle joint [57].

Furthermore, for the analysis of the coordination of body segments, maximum segment rotation, maximum angular velocity, range of the angular velocity and parameters of timing will be calculated. Similar measures have previously been published $[58,59]$.

Dynaport Hybrid Sway parameters, such as mediolateral and anteroposterior postural sway length and velocity will be calculated for analysis of postural stability during static standing [60, 61].

Parameters related to dynamic stability in walking including stride-time, stride-time variability and stability of gait (LDS) will be generated [62, 63].

\section{Prospective falls assessment}

All participants will receive a prospective fall protocol (expert consensus; http://farseeingresearch.eu). They will be asked to document any fall during the next year. A study nurse will remind the participants once a month via telephone call to fill in the protocol, and monitor the fall protocols completed for each fall so far. After 1 year, the fall protocol of each subject will be collected.

\section{Discussion}

Reduction of falls frequency in an ageing society is a major aim of health care. Exact mechanisms of suspected causal factors like VOR suppression deficits are yet unknown and a systematic analysis of these mechanisms is urgently needed. This study protocol describes an experimental setup allowing the analysis of simultaneously assessed eye, head and body movements. It helps to further understand the association of VOR suppression deficit and falls. Prospective falls assessment within this study will eventually enable a calculation of the predictive validity of the clinical and biomechanical variables.

\section{Study strengths and limitations}

To the best of our knowledge, this is the first study to assess high-frequency VOR, VOR suppression, balance and gait simultaneously under quantitative experimental conditions.

Further, it includes neurogeriatric cohorts at high risk for falls. Patients will be selected by movement disorders specialists at the Department of Neurodegeneration, Center for Neurology, University Hospital Tuebingen.

Nevertheless, our study has some limitations. First, the sample size is relatively small. However, participants will be tested extensively in a very standardized protocol. This setting will allow the extraction of relevant information for future, more hypothesis-driven studies.

Although our setup approaches natural conditions by extending the examination of VOR-suppression to standing and walking, it remains artificial insofar that subjects walk on a treadmill in a sparse and dark environment. In principle, translation of our results into real-life outdoor conditions might be considered in future experiments. However, we believe that this setup allows precise and detailed analysis of the association of VOR suppression deficits and balance and gait measures and leads to a better understanding of the interaction between the oculomotor and the locomotor systems.

\section{Conclusions}

Our protocol uniquely combines sound methods in analyzing mechanistic aspects of the association of oculoand locomotion and a clinically relevant prospective falls assessment in ageing and neurodegenerative disease. We will investigate established and novel markers of fall risk related to VOR suppression deficit, gait and balance using a new setup with high technical standard. The results will help to better understand the mechanisms of falls and to design new therapeutic interventions.

\section{Additional files}

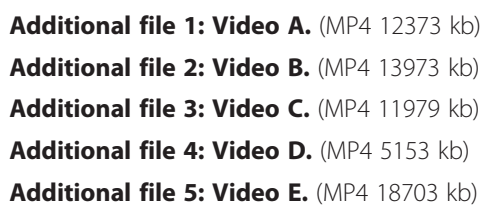

Abbreviations

ADS: Allgemeine Depressions Skala; CoM: Extrapolated center of mass; DGI: Dynamic Gait Index; FES-I: Short Form of the Falls Efficacy Scale-International; 
HIT: Head-impulse test; LLFDI: Late-Life Function and Disability Instrument; LDS: Local dynamic stability; LED: Light-emitting diodes; MoCA: Montreal Cognitive Assessment; NPI: Neuropsychiatric Inventory; PSP: Progressive Supranuclear Palsy; PSP-RS: Progressive Supranuclear Palsy Rating Scale; PD: Parkinson's disease; TMT A + B: Trail Making Test parts A and B; UMSARS: Unified Multiple System Atrophy Rating Scale; MDS-UPDRS: Unified Parkinson's Disease Rating Scale, Movement Disorders Society; VOR: Vestibuloocular reflex; VOR S: The suppression of the vestibulo-ocular reflex; VOR gain: Eye velocity/head velocity; VICON: 3-D motion capture system.

\section{Competing interests}

ES acts as an unpaid consultant and has received funding for travel from Interacoustics, distributor of EyeSeeCam. He is the general manager and a shareholder of EyeSeeTec $\mathrm{GmbH}$, manufacturer of EyeSeeCam.

\section{Authors' contributions}

Conception and design: KS, JK, JP, MSy, DB, WM, CB. Acquisition, analysis or interpretation of data: KS, DJM, JK, LS, EAFI, MS, UL, MM, SKC, MAH, KB, IW ES, UI, WM, CB. Drafted the manuscript: KS, DJM, JK, JP, WM. Critical revision of the manuscript for important intellectual content: LS, EAFI, MS, UL, MM SKC, MAH, KB, IW, MSy, ES, UI, DB, CB. All authors read and approved the final manuscript.

\section{Authors' information}

Not applicable.

\section{Acknowledgements}

This study is funded by grants from the Forschungskolleg Geriatrie of the Robert-Bosch-Foundation Stuttgart, Germany (to K.S. and M.Sy.) and in part by the German Federal Ministry of Education and Research (Grant 01 EO 0901).

We thank Mr. Alexander Konradi for support in graphic design. The authors acknowledge support by Deutsche Forschungsgemeinschaft and Open Access Publishing Fund of University of Tübingen.

\section{Author details}

'Department of Neurodegeneration, Hertie Institute for Clinical Brain Research, University of Tuebingen, Tuebingen, Germany. ${ }^{2}$ Department of Geriatrics and Clinic of Geriatric Rehabilitation, Robert-Bosch-Hospital, Stuttgart, Germany. ${ }^{3}$ Department of Cognitive Neurology, Hertie Institute for Clinical Brain Research, University of Tuebingen, Tuebingen, Germany. ${ }^{4}$ Clinic for Neurology, University Hospital Zurich, Zurich, Switzerland. ${ }^{5}$ Institute of Epidemiology and Medical Biometry, Ulm University, Ulm, Germany. ${ }^{6}$ Department of Neuroscience, Norwegian University of Science and Technology, Trondheim, Norway. ${ }^{7}$ German Research Center for Neurodegenerative Diseases (DZNE), University of Tuebingen, Tuebingen, Germany. ${ }^{8}$ Institute of Medical Technology, Brandenburg University of Technology Cottbus -Senftenberg, Cottbus, Germany.

\section{Received: 13 August 2015 Accepted: 29 September 2015}

\section{Published online: 09 October 2015}

\section{References}

1. Nations U: World Population Ageing 2013. In. Edited by Department of Economic and Social Affairs PD; 2013.

2. Ganz DA, Bao Y, Shekelle PG, Rubenstein LZ. Will my patient fall? JAMA. 2007;297(1):77-86

3. Bloem BR, Steijns JA, Smits-Engelsman BC. An update on falls. Curr Opin Neurol. 2003;16(1):15-26

4. Hollands MA, Marple-Horvat DE. Coordination of eye and leg movements during visually guided stepping. J Mot Behav. 2001;33(2):205-16.

5. Glasauer S, Schneider E, Jahn K, Strupp M, Brandt T. How the eyes move the body. Neurology. 2005;65(8):1291-3

6. Collins CJ, Barnes GR. Independent control of head and gaze movements during head-free pursuit in humans. J Physiol. 1999;515(Pt 1):299-314.

7. Roy JE, Cullen KE. Vestibuloocular reflex signal modulation during voluntary and passive head movements. J Neurophysiol. 2002:87(5):2337-57.

8. Einhauser W, Schumann F, Bardins S, Bartl K, Boning G, Schneider E, et al. Human eye-head co-ordination in natural exploration. Network. 2007;18(3):267-97.
9. Thier $\mathrm{P}, \mathrm{llg}$ UJ. The neural basis of smooth-pursuit eye movements. Curr Opin Neurobiol. 2005;15(6):645-52.

10. Leigh R, Zee D. The neurology of eye movements. 2006.

11. Roy JE, Cullen KE. Dissociating self-generated from passively applied head motion: neural mechanisms in the vestibular nuclei. J Neurosci. 2004;24(9):2102-11.

12. Sparks DL. The brainstem control of saccadic eye movements. Nat Rev Neurosci. 2002;3(12):952-64.

13. Barnes GR, Paige GD. Anticipatory VOR suppression induced by visual and nonvisual stimuli in humans. J Neurophysiol. 2004:92(3):1501-11.

14. Cullen KE, Huterer M, Braidwood DA, Sylvestre PA. Time course of vestibuloocular reflex suppression during gaze shifts. J Neurophysiol. 2004;92(6):3408-22.

15. Roy JE, Cullen KE. A neural correlate for vestibulo-ocular reflex suppression during voluntary eye-head gaze shifts. Nat Neurosci. 1998;1(5):404-10.

16. Tabak S, Smeets JB, Collewijn H. Modulation of the human vestibuloocular reflex during saccades: probing by high-frequency oscillation and torque pulses of the head. J Neurophysiol. 1996;76(5):3249-63.

17. Fuchs AF, Ling L, Phillips JO. Behavior of the position vestibular pause (PVP) interneurons of the vestibuloocular reflex during head-free gaze shifts in the monkey. J Neurophysiol. 2005;94(6):4481-90.

18. Hacke KPW. Neurologie. 2006

19. Land MF. Eye movements and the control of actions in everyday life. Prog Retin Eye Res. 2006;25(3):296-324

20. Chen LL. Head movements evoked by electrical stimulation in the frontal eye field of the monkey: evidence for independent eye and head control. J Neurophysiol. 2006;95(6):3528-42.

21. Baloh RW, Jacobson KM, Socotch TM. The Effect of Aging on VisualVestibuloocular Responses. Exp Brain Res. 1993;95(3):509-16.

22. Demer JL. Effect of aging on vertical visual tracking and visual-vestibular interaction. J Vestibular Res. 1994:4(5):355-70.

23. Paige GD. Senescence of human visual-vestibular interactions: smooth pursuit, optokinetic, and vestibular control of eye movements with aging. Exp Brain Res. 1994;98(2):355-72.

24. Rascol O, Clanet M, Montastruc JL, Simonetta M, Soulier-Esteve MJ, Doyon B, et al. Abnormal ocular movements in Parkinson's disease. Evidence for involvement of dopaminergic systems. Brain. 1989:112(Pt 5):1193-214.

25. White OB, Saint-Cyr JA, Sharpe JA. Ocular motor deficits in Parkinson's disease. I. The horizontal vestibulo-ocular reflex and its regulation. Brain. 1983;106(Pt 3):555-70.

26. Halmagyi GM, Gresty MA. Clinical signs of visual-vestibular interaction. J Neurol Neurosurg Psychiatry. 1979;42(10):934-9.

27. Duvoisin RC, Golbe LI, Lepore FE. Progressive supranuclear palsy. Can 」 Neurological Sci Le J Canadien des sciences neurologiques. 1987;14(3 Suppl):547-54.

28. Di Fabio RP, Zampieri C, Tuite P, Konczak J. Association between vestibuloocular reflex suppression during smooth movements of the head and attention deficit in progressive supranuclear palsy. Movement Disorders. 2006;21 (7):910-5.

29. Paquette $C$, Fung J. Old age affects gaze and postural coordination. Gait Posture. 2011;33(2):227-32.

30. Greany JF, Di Fabio RP. Saccade to stepping delays in elders at high risk for falling. Aging Clin Exp Res. 2008;20(5):428-33.

31. Di Fabio RP, Emasithi A, Greany JF, Paul S. Suppression of the vertical vestibulo-ocular reflex in older persons at risk of falling. Acta Otolaryngol. 2001;121(6):707-14.

32. Di Fabio RP, Greany JF, Emasithi A, Wyman JF. Eye-head coordination during postural perturbation as a predictor of falls in community-dwelling elderly women. Arch Phys Med Rehabil. 2002;83(7):942-51.

33. Kerber KA, Ishiyama GP, Baloh RW. A longitudinal study of oculomotor function in normal older people. Neurobiol Aging. 2006;27(9):1346-53.

34. Stoffregen TA, Bardy BG, Bonnet CT, Hove P, Oullier O. Postural sway and the frequency of horizontal eye movements. Mot Control. 2007;11(1):86-102.

35. Young WR, Hollands MA. Evidence for age-related decline in visuomotor function and reactive stepping adjustments. Gait Posture. 2012;36(3):477-81.

36. Di Fabio RP, Zampieri C, Tuite P. Gaze-shift strategies during functional activity in progressive supranuclear palsy. Exp Brain Res. 2007;178(3):351-62.

37. Litvan I, Agid Y, Calne D, Campbell G, Dubois B, Duvoisin RC, et al. Clinical research criteria for the diagnosis of progressive supranuclear palsy (Steele-Richardson-Olszewski syndrome): report of the NINDS-SPSP international workshop. Neurology. 1996;47(1):1-9. 
38. Hughes AJ, Daniel SE, Kilford L, Lees AJ. Accuracy of clinical diagnosis of idiopathic Parkinson's disease: a clinico-pathological study of 100 cases. J Neurol Neurosurg Psychiatry. 1992;55(3):181-4.

39. Hoehn MM, Yahr MD. Parkinsonism: onset, progression and mortality. Neurology. 1967;17(5):427-42

40. Groll DL, To T, Bombardier C, Wright JG. The development of a comorbidity index with physical function as the outcome. J Clin Epidemiol. 2005;58(6):595-602.

41. Kempen GI, Yardley L, van Haastregt JC, Zijlstra GA, Beyer N, Hauer K, et al. The Short FES-l: a shortened version of the falls efficacy scale-international to assess fear of falling. Age Ageing. 2008;37(1):45-50.

42. Denkinger MD, Igl W, Coll-Planas L, Bleicher J, Nikolaus T, Jamour M. Evaluation of the short form of the late-life function and disability instrument in geriatric inpatients-validity, responsiveness, and sensitivity to change. J Am Geriatr Soc. 2009;57(2):309-14

43. Nasreddine ZS, Phillips NA, Bedirian V, Charbonneau S, Whitehead V, Collin I, et al. The Montreal Cognitive Assessment, MoCA: a brief screening tool for mild cognitive impairment. J Am Geriatr Soc. 2005;53(4):695-9.

44. Corrigan JD, Hinkeldey NS. Relationships between parts A and B of the Trail Making Test. J Clin Psychol. 1987;43(4):402-9.

45. Hautzinger M, Bailer M. Allgemeine Depressions Skala. Manual. Göttingen, Beltz: Manual; 1993.

46. Cummings JL, Mega M, Gray K, Rosenberg-Thompson S, Carusi DA Gornbein J. The Neuropsychiatric Inventory: comprehensive assessment of psychopathology in dementia. Neurology. 1994:44(12):2308-14

47. Wenning GK, Tison F, Seppi K, Sampaio C, Diem A, Yekhlef F, et al. Development and validation of the Unified Multiple System Atrophy Rating Scale (UMSARS). Movement Disorders. 2004;19(12):1391-402.

48. Golbe LI, Ohman-Strickland PA. A clinical rating scale for progressive supranuclear palsy. Brain. 2007;130(Pt 6):1552-65

49. Goetz CG, Fahn S, Martinez-Martin P, Poewe W, Sampaio C, Stebbins GT, et al. Movement Disorder Society-sponsored revision of the Unified Parkinson's Disease Rating Scale (MDS-UPDRS): Process, format, and clinimetric testing plan. Movement Disorders. 2007;22(1):41-7.

50. Shumway-Cook A. Theory and practical applications, Motor Control. 1995.

51. Halmagyi GM, Black RA, Thurtell MJ, Curthoys IS. The human horizontal vestibulo-ocular reflex in response to active and passive head impulses after unilateral vestibular deafferentation. Ann N Y Acad Sci. 2003;1004:325-36

52. Holland PW, Welsch RE. Robust Regression Using Iteratively Reweighted Least-Squares, vol. A6. 1977

53. Howcroft J, Kofman J, Lemaire ED. Review of fall risk assessment in geriatric populations using inertial sensors. J Neuroeng Rehabil. 2013;10(1):91.

54. Rispens SM, van Schooten KS, Pijnappels M, Daffertshofer A, Beek PJ, van Dieen $\mathrm{JH}$. Identification of fall risk predictors in daily life measurements: gait characteristics' reliability and association with self-reported fall history. Neurorehabil Neural Repair. 2015;29(1):54-61.

55. Oddsson LI, Wall C, McPartland MD, Krebs DE, Tucker CA. Recovery from perturbations during paced walking. Gait Posture. 2004;19(1):24-34.

56. Suptitz F, Karamanidis K, Moreno Catala M, Bruggemann GP. Symmetry and reproducibility of the components of dynamic stability in young adults at different walking velocities on the treadmill. J Electromyogr Kinesiol. 2012;22(2):301-7.

57. Hof AL, Gazendam MG, Sinke WE. The condition for dynamic stability. J Biomech. 2005;38(1):1-8.

58. Hollands MA, Ziavra NV, Bronstein AM. A new paradigm to investigate the roles of head and eye movements in the coordination of whole-body movements. Exp Brain Res. 2004;154(2):261-6.

59. Cinelli M, Patla A, Stuart B. Age-related differences during a gaze reorientation task while standing or walking on a treadmill. Exp Brain Res. 2008;185(1):157-64.

60. Doheny EP, McGrath D, Greene BR, Walsh L, McKeown D, Cunningham C, et al. Displacement of centre of mass during quiet standing assessed using accelerometry in older fallers and non-fallers. Conference Proc. 2012:2012:3300-3.

61. Greene BR, McGrath D, Walsh L, Doheny EP, McKeown D, Garattini C, et al. Quantitative falls risk estimation through multi-sensor assessment of standing balance. Physiol Meas. 2012;33(12):2049-63.
62. Toebes MJ, Hoozemans MJ, Furrer R, Dekker J, van Dieen JH. Local dynamic stability and variability of gait are associated with fall history in elderly subjects. Gait Posture. 2012;36(3):527-31.

63. van Schooten KS, Sloot LH, Bruijn SM, Kingma H, Meijer OG, Pijnappels M, et al. Sensitivity of trunk variability and stability measures to balance impairments induced by galvanic vestibular stimulation during gait. Gait Posture. 2011;33(4):656-60

\section{Submit your next manuscript to BioMed Central and take full advantage of:}

- Convenient online submission

- Thorough peer review

- No space constraints or color figure charges

- Immediate publication on acceptance

- Inclusion in PubMed, CAS, Scopus and Google Scholar

- Research which is freely available for redistribution 